\title{
Novel Setup for High Performance Simultaneous 3D EBSD and 3D EDS Acquisition
}

\author{
Rostislav Váňa ${ }^{1}$, Jiř́i Dluhoš ${ }^{1}$, Lukáš Hladík ${ }^{1}$, John Lindsay², Jenny Goulden ${ }^{2}$ \\ 1. TESCAN Brno, s.r.o, R\&D Applications, Brno, Czech Republic \\ 2. Oxford Instruments, Nanoanalysis, High Wycombe, United Kingdom
}

A high resolution scanning electron microscope (SEM) combined with a focused ion beam (FIB) is used for high precision tomography using FIB slicing and SEM observation of the slice. The FIB-SEM can be further equipped with analytical methods like energy-dispersive X-ray spectrometer (EDS) for the determination of elemental composition or electron backscattered diffraction analyzer (EBSD) to observe crystal orientation. This multi-modal method results in three-dimensional microanalysis, i.e. combined 3D EDS and 3D EBSD. These techniques are already well established with FIB-SEM tomography [1].

However, the speed and comprehensiveness of data acquisition has been greatly improved over the past few years. In this work, we present a novel setup for rapid acquisition of simultaneous 3D EBSD and 3D EDS using an integrated solution comprising a novel TESCAN Xe $\mathrm{e}^{+}$Plasma FIB-SEM and the Oxford Instruments AZtec 3D Microanalysis system.

The long-standing practical limit of FIB-SEM tomography at most to under 100 micrometers was based on the limitations to the maximum beam current of conventional $\mathrm{Ga}^{+} \mathrm{FIB}$ systems. With the wide availability of high performance $\mathrm{Xe}^{+}$plasma $\mathrm{FIB}$, large volumes with each dimension in the hundreds of microns can now be reconstructed easily within a few hours $[2,3]$. The latest generation of the HR i-FIB column in combination with the proprietary TRUE X-sectioning milling technique enables curtain-free cross-sectioning even at maximum FIB beam currents (see Fig. 1).

The "static sample" approach has been recognized as the optimal technique for high speed and high precision 3D acquisition [1]. The unique geometrical configuration of analytics patented by TESCAN [4] allows simultaneous 3D EDS and EBSD acquisition with no sample movement required for FIB milling and data collection (see Fig. 2).

A novel setup for 3D microanalysis from Oxford Instruments comprising the high speed EDS detector X$\operatorname{Max}^{\mathrm{N}}$ and the EBSD camera Nordlys $\mathrm{Max}^{3}$ was used. The new capabilities of AZtec 3D Microanalysis allow simultaneous EDS and EBSD data collection at speeds up to 1580 points per second followed by real-time TruPhase ${ }^{\mathrm{TM}}$ identification using both EDS and EBSD data (see Fig. 3). The EDS results are aided by the superior AZtec TruMap ${ }^{\mathrm{TM}}$ deconvolution and quantitative mapping.

Processing large datasets consisting of 3D SEM images, EBSD and EDS data is another significant challenge. Python scripting with its computational power adequate for processing large multi-channel datasets was used for data management.

References:

[1] S. Zaefferer, Book of abstracts EMAS Workshop, p. 123-136, (2009)

[2] T. Hrnčír, et al, Microsc.Microanal.19, (Suppl 2), p. 860-862, (2013) 
[3] T. Hrnčír, L. Hladík, Proceedings from the 39th International Symposium for Testing and Failure Analysis, (2013), p. 27

[4] Patent CZ 301692, (2009)
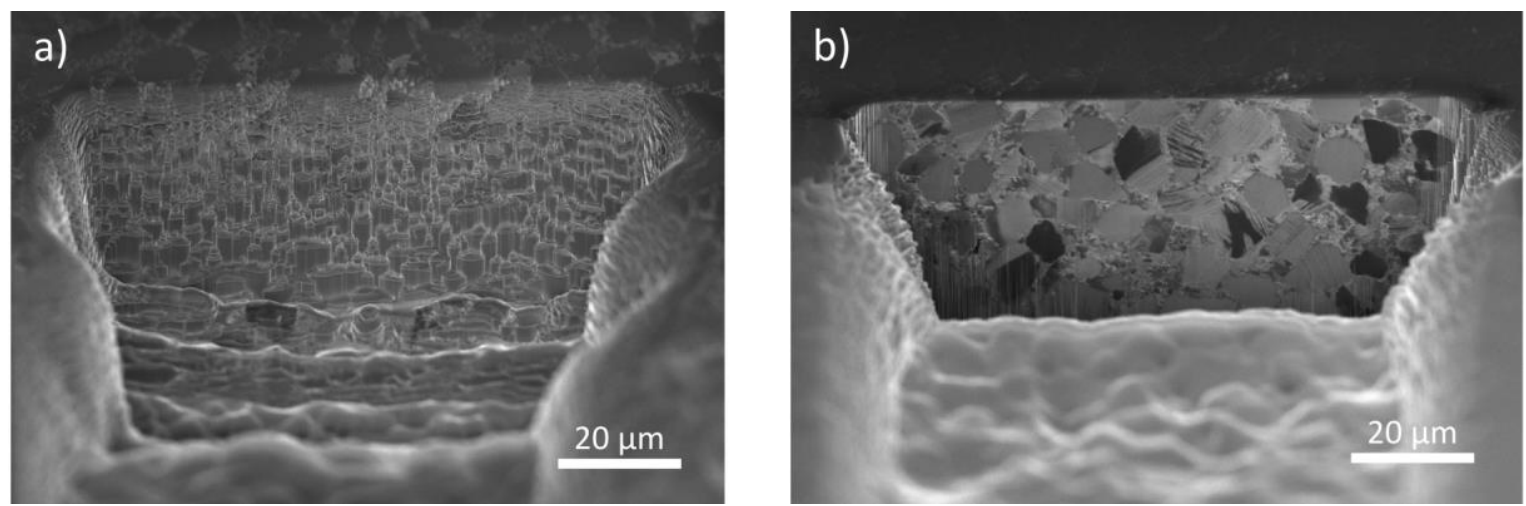

Figure 1. Example of TRUE X-sectioning technique on SiC composite. a) Milling at high current with no curtaining reduction technique. b) curtain-free, polished cross section using TRUE X-sectioning technique .
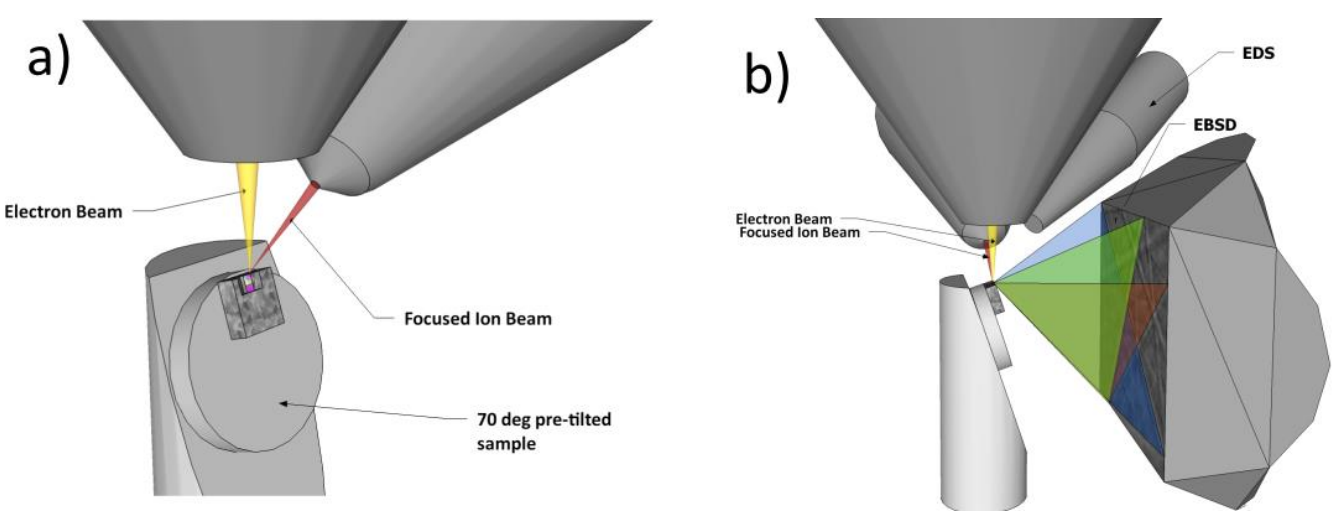

Figure 2. Static acquisition geometry on the TESCAN FIB-SEM. a) Sample on a pre-tilted holder. b) Configuration allowing simultaneous 3D EBSD and 3D EDS acquisition with no sample movement.
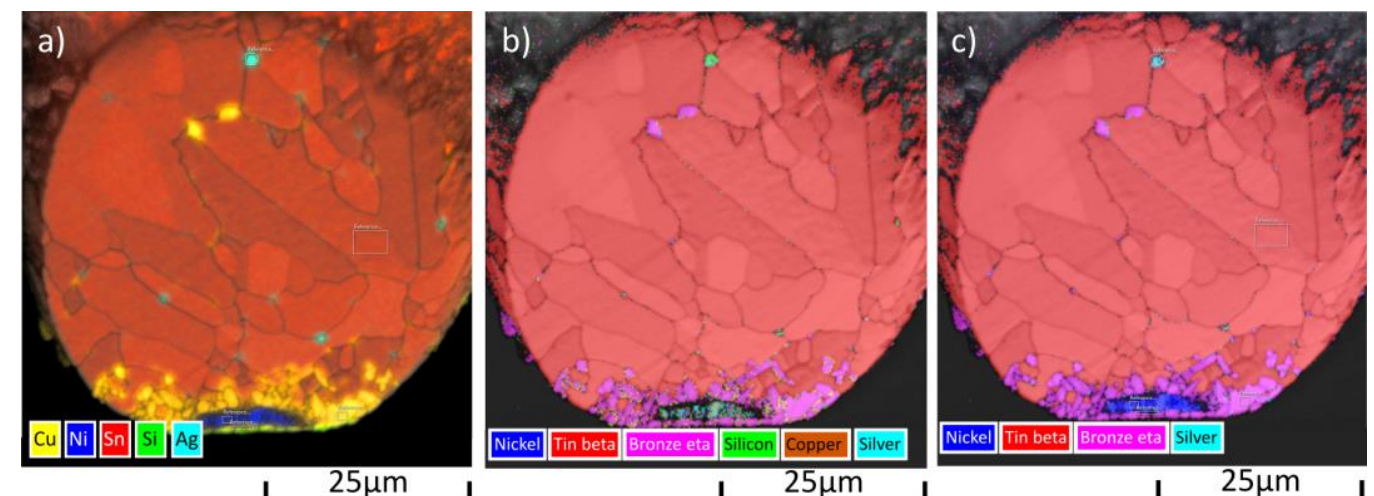

Figure 3. Comparison of different phase analysis techniques a) EDS map using TruMap ${ }^{\mathrm{TM}}$ deconvolution, b) EBSD identification without EDS correction and c) TruPhase ${ }^{\mathrm{TM}}$ mapping result with reference spectra used for phase identification correction on phases with a similar crystal lattice. 